\title{
Akteure im Rahmen des Perspektivenwechsels: Ergebnisse einer Analyse von geographiedidaktischen Unterrichtsmaterialien
}

\author{
"d.vasiljuk@uni-koeln.de, Institut für Geographiedidaktik, Universität zu Köln, ( $\triangle$ korresp. Autorin) \\ **alexandra.budke@uni-koeln.de, Institut für Geographiedidaktik, Universität zu Köln
}

eingereicht am: 05.01.2021, akzeptiert am: 27.03.2021

\begin{abstract}
Im Artikel wird der Frage nachgegangen, welche Akteure ${ }^{1}$ im Kontext des Perspektivenwechsels in geographiedidaktischen Unterrichtsmaterialien verwendet werden. Hierfür werden die Unterrichtsmaterialien hinsichtlich Akteursebene, Akteursart und Akteurstyp analysiert. Anschließend werden die verschiedenen Potentiale und Hürden bei der Materialentwicklung für die Umsetzung des Perspektivenwechsels im Geographieunterricht diskutiert.
\end{abstract}

Keywords: Akteure, Akteursanalyse, Perspektivenwechsel, Multiperspektivität

\section{Actors in the context of perspective-taking: results of an analysis of geography lesson concepts}

The article investigates which actors are used in terms of perspective-taking in geography lesson concepts. Therefore, lesson concepts are analysed regarding scales, categories, and types of the respective actors. Afterwards, several potentials and challenges are discussed referring to the lesson concept development for implementing perspective-taking in geography lessons.

Keywords: actors, actor analysis, perspective-taking, multiperspectivity

\section{Einleitung}

Dem Global Risk Report zufolge muss sich die Gesellschaft in den folgenden Jahrzehnten verschiedenen Megatrends und Risiken stellen (vgl. World Economic Forum 2017), die aufgrund von unvereinbaren Zielvorstellungen der beteiligten Akteure ein sehr hohes gesellschaftliches Konfliktpotential aufweisen können (vgl. Bonacker 2009: 181). Dazu gehören konfliktträchtige Themenkomplexe wie der Klimawandel und Umweltdegradation, zunehmende Migration sowie zunehmender Nationalismus und Umbrüche politischer Machtverhältnisse (vgl. World Economic Forum 2017: 66 ff.). Auch im Geographieunterricht werden die Entwicklungen dieser Konflikte behandelt (vgl. DGFG 2017: 5 ff.).

Aus Gründen der vielzähligen Wortbedeutung (Individuen, Gruppen, Organisationen) und der Verwendung des Wortes in der ökonomischen Fachwissenschaft, wird in diesem Beitrag bei dem Wort „Akteur*innen“ die Form „Akteure“ verwendet. Der Begriff gilt im Sinne der Gleichbehandlung für alle Geschlechter. Wir sind uns der Problematik des Begriffes bewusst. Die verkürzte Sprachform hat redaktionelle Gründe und beinhaltet keine Wertung.
Wenn Konflikte im Geographieunterricht dargestellt werden, muss beachtet werden, dass sie sich durch verschiedene Perspektiven und Interessen unterschiedlicher Akteure begründen, die ihre räumliche und soziale Umwelt konstruieren, interpretieren und repräsentieren (vgl. Reuber 2000: 41). Als Konsequenz daraus existiert im Sinne des Konstruktivismus keine objektive Konfliktwirklichkeit, „sondern nur eine Vielfalt miteinander konkurrierender Sichtweisen“" (Reuber 2001: 90). Mithilfe des Perspektivenwechsels kann diese Vielfalt aufgezeigt werden, sodass Schüler*innen zunächst den Konflikt aus den Sichtweisen verschiedener Akteure betrachten, um ihn anschließend in seiner Komplexität erfassen und beurteilen zu können (vgl. DGFG 2017: 13 ff.).

Es können allerdings bei der Darstellung des Konflikts verschiedene didaktische Hürden entstehen, die bei der Konzeption der Unterrichtsmaterialien beachtet werden sollten. Zum einen sollten Akteure für den Perspektivenwechsel ausgewählt werden, die den Schüler*innen ein strittiges Thema in ihrer Komplexität und Kontroversität widerspiegeln (vgl. Wehling 1977). Zum anderen darf keine zu starke didaktische 
Reduktion erfolgen, durch die ein Konflikt und die damit einhergehenden, oftmals unvereinbaren Zielvorstellungen und Schwierigkeiten hinsichtlich einer Lösungsfindung nicht mehr aufgezeigt werden können (vgl. Kiehl \& Schnerch 2018). Ein theoretisches Verständnis von Akteuren und deren Typologie könnte daher bei der Konzeption der Unterrichtsmaterialien hilfreich sein, um eine gezielte Auswahl der Akteure treffen und den Konflikt darstellen zu können.

In bisherigen Arbeiten wurden Akteure in raumbezogenen Konflikten mithilfe von Diskurs- und Argumentationsanalysen untersucht (vgl. Kuckuck 2014: 39 ff.) oder es wurden in geographiedidaktischen Materialien u. a. die verschiedenen Perspektiven des Konflikts analysiert (vgl. Stöber 2011; Kuckuck 2014: $83 \mathrm{ff}$.). Bislang wurden die Akteure in Unterrichtsmaterialien allerdings nicht mithilfe einer theoretisch fundierten Klassifikation erfasst, um sie anschließend analysieren zu können. Auch wurden die für den Perspektivenwechsel gewählten Akteure in den Unterrichtsmaterialien weder untersucht noch die didaktischen Herausforderungen diskutiert.

Aus diesem Grund wurde eine Untersuchung von 28 geographiedidaktischen Artikeln aus insgesamt vier geographiedidaktischen Praxiszeitschriften aus dem deutschsprachigen Raum vorgenommen.

Der Artikel zielt daher darauf ab, folgende Fragen zu beantworten:

- Welche Akteure werden im Rahmen des Perspektivenwechsels für die Darstellung des Konflikts gewählt?

- Inwiefern zeigen die gewählten Akteure die Komplexität des jeweiligen Konflikts auf?

Um die von den Autor*innen gewählten Akteure in den geographiedidaktischen Unterrichtsmaterialien genauer untersuchen zu können, wurde eine eigene Klassifikation auf Basis verschiedener Ansätze entwickelt. Daher geht der Artikel zunächst kurz auf ausgewählte theoretische Konzepte ein, um herauszustellen, wie Akteure definiert und klassifiziert werden können. Danach werden das methodische Vorgehen der qualitativen Inhaltsanalyse und die zentralen Ergebnisse präsentiert und diskutiert.

\section{Klassifikation der Akteure}

Die verschiedenen theoretischen Auseinandersetzungen mit Akteuren verfolgen je nach Schwerpunkt und Fachdisziplin unterschiedliche Erkenntnisinteressen, wie u. a. das Verhalten der Akteure zu erklären, Beziehungen der Akteure sowie deren Macht darzustellen oder die Interaktion von Akteuren anhand von verschiedenen Parametern zu untersuchen (vgl. Mitchell et al. 1997; Dahrendorf 2010). Ein Akteur zeichnet sich dabei zumeist in Anlehnung an Weber (vgl. 1980) und Schimank (vgl. 2011) durch bestimmte Präferenzen, Fähigkeiten und Wahrnehmungen aus und ist ein Handelnder, dessen Handlungsvoraussetzung eine Gruppe ist. Eine Gruppe kann wiederum verschiedenen Disziplinen wie der Politik, Wissenschaft, Wirtschaft oder Zivilgesellschaft zugeordnet werden. Dabei können aber nur Individuen Träger von sinnhaft orientiertem Handeln sein und im Namen sowie im Interesse von anderen Personen, Gruppen oder Organisationen handeln.

Zwar gibt es zahlreiche theoretische Auseinandersetzungen, die sich mit Akteuren befassen, für die Analyse musste allerdings eine eigene Klassifikation entwickelt werden. Bestehende Klassifikationen waren für die Untersuchung des geographiedidaktischen Materials nicht gänzlich geeignet, da diese entweder Aspekte beinhalten, die für eine geographiedidaktische Auseinandersetzung nicht zielführend genug oder irrelevant waren. Die eigene Klassifikation wurde daher aus verschiedenen theoretischen Konzepten abgeleitet. Hierbei wurden Konzepte gewählt, die Akteure im Rahmen eines Konflikts definieren und mittels einer Klassifikation analysieren.

In diesem Kapitel werden daher zunächst die ausgewählten theoretischen Konzepte aus den Fachdisziplinen Politische Ökologie, Politikwissenschaft und Geographie kurz vorgestellt, die vor allem als Grundlage für die Entwicklung der eigenen Klassifikation dienten. Danach wird die entwickelte Klassifikation der Akteure vorgestellt. Um die Wahl der Akteure und deren Auswirkungen für den Geographieunterricht diskutieren zu können, werden anschließend auch mögliche Hürden bei der didaktischen Reduktion aufgezeigt, die bei der Unterrichtskonzeption entstehen können und bei deren Bewältigung die Klassifikation der Akteure von Nutzen sein kann.

\subsection{Theoretische Grundlage}

Im akteurszentrierten Institutionalismus steht die „Steuerung und Selbstorganisation auf der Ebene ganzer gesellschaftlicher Teilbereiche" im Vordergrund, bei der die Akteure genauer betrachtet werden (Maynatz \& Scharpf 1995: 49 ff.). Aufbauend auf diesem Ansatz können nach dem Politikwissenschaftler Scharpf (vgl. 2000: 95 ff.) Akteure in individuelle, aggregierte und komplexe Akteure unterteilt werden, um u. a. Konfliktlösungsmechanismen analysieren zu können. Aggregierte Akteure setzen sich aus mehreren individuellen Akteuren zusammen, die ähnliche Präferenzen haben und daher gleichgerichtet handeln, allerdings genauso wie individuelle Akteure meistens keine strategischen Entscheidungen treffen können. Komplexe Akteure entsprechen dagegen einer Men- 
ge von mehreren individuellen Akteuren, welche die Fähigkeit zum intentionalen Handeln aufweisen. Zudem ist deren Wahrscheinlichkeit strategische Entscheidungen zu treffen höher als bei aggregierten oder individuellen Akteuren. Komplexe Akteure können weiter in kollektive (z. B. Bewegungen, Verbände, strategische Allianzen) und korporative Akteure (z. B. Gewerkschaften und Unternehmen) unterteilt werden. Während kollektive Akteure von den Präferenzen ihrer Mitglieder weitestgehend abhängig sind, können korporative Akteure Entscheidungen treffen, ohne dabei die Präferenzen und Ziele ihrer Mitglieder berücksichtigen zu müssen.

Ein relevantes Kriterium bei der Akteursklassifikation nach Scharpf (vgl. 2000: 95 ff.) ist die Differenzierung der Akteure nach ihrer Zusammensetzung (ein Individuum gegenüber mehreren Individuen) und ihrer Fähigkeit zum intentionalen Handeln (individuell und aggregiert gegenüber komplex). Dadurch kann u. a. auch aufgezeigt werden, dass Akteure unterschiedliche Handlungsfähigkeiten aufweisen.

In der politischen Ökologie werden vor allem die Interessen, Beziehungen und Machtverhältnisse der jeweiligen Akteure innerhalb eines politisch-ökologischen Konflikts analysiert. Bei der Analyse erfolgt die Unterteilung zwischen lokalen Akteuren (place-based actors) sowie nationalen und internationalen Akteuren (non-place-based actors), da unterschiedliche räumliche Handlungsebenen betroffen sein können (vgl. Robbins 2012: 45). Während place-based actors (z. B. regionale Bäuerinnen*Bauern) einem ökologischen Konflikt direkt ausgesetzt sind und lokal handeln, sind nonplace-based actors (z.B. Entscheidungsträger*innen verschiedener Institutionen) nicht unmittelbar von diesem Konflikt betroffen und können über die lokale Ebene hinaus handeln (vgl. Bryant \& Bailey 1997; Bauriedl 2016: 345).

Herausragendes Merkmal der Unterteilung von Bryant \& Bailey (vgl. 1997) sowie Bauriedl (vgl. 2016: 345) für das Fach Geographie ist die Maßstäblichkeit der Akteure. Es wird hierbei für die Analyse einerseits unterschieden auf welcher räumlichen Ebene die Akteure vom Konflikt betroffen sind, andererseits auf welcher räumlichen Ebene sie handeln können.

In der politischen Geographie werden die Zusammenhänge zwischen Macht und Raum analysiert. Dabei stehen im handlungsorientierten Ansatz der politischen Geographie die Handlungen der Akteure im Fokus (vgl. Reuber 2000, 2001). Eine handlungsorientierte Untersuchung verfolgt dabei das Ziel, „subjektive Raumkonzepte und Konfliktsichten im Wechselspiel subjektiver Interessen, gesellschaftspolitischer Strukturen und physisch-materieller Rahmenbedingungen zu verstehen" (Reuber 2001: 90). Ausgangspunkt ist somit ein konstruktivistisches Weltbild (vgl.
Reuber 2001: 81). Um der Frage der Macht der Akteure in Raumnutzungskonflikten nachgehen und diese analysieren zu können, muss der Begriff zunächst definiert werden. Reuber (vgl. 2001: 84 ff.) schlägt hierbei ein Dreisäulen-Konzept der Macht vor, das in autoritative Ressourcen (z. B. Netzwerke, politische Position im Entscheidungssystem), allokative Ressourcen (z. B. finanzielle Potentiale, Grund und Boden) und in individuelle Komponenten (z. B. persönliches Charisma, Durchsetzungsstärke) unterteilt wird. Die autoritativen und allokativen Ressourcen entsprechen dabei einer strukturellen Komponente. Anzumerken ist, dass die strukturellen und individuellen Komponenten bei der Handlung eines Akteurs miteinander verknüpft sind und in Wechselwirkung zueinander stehen.

Im Unterschied zu den ausgeführten Konzepten aus der Politikwissenschaft und Politischen Ökologie steht bei der Analyse der Akteure nach Reuber (vgl. 2001) sowohl die subjektive Konstruktion von raumbezogenen Konflikten als auch die Analyse ihrer Macht im Vordergrund. Für die Klassifikation sind diese Aspekte insofern wichtig, da sie aufzeigen, dass es keine objektive Konfliktwirklichkeit gibt und vorhandene Ressourcen eines Akteurs darüber entscheiden können, ob er seine Interessen durchsetzen kann.

\subsection{Vorstellung der entwickelten Klassifikation für die eigene Untersuchung}

Für die Analyse der geographiedidaktischen Artikel wird der Begriff des „Akteurs“ auf Grundlage der dargelegten theoretischen Ansätze wie folgt klassifiziert: Sichtweisen sind an Akteure gebunden (vgl. Reuber 2000: 41). Jede Sichtweise eines Akteurs kann dabei einer Akteursebene, somit einer räumlichen Maßstabebene (lokal, regional, national oder global) zugeordnet werden (vgl. Bryant \& Bailey 1997; Bauriedl 2016). Die Akteursebene gibt somit i.e.S. wieder, auf welchem Maßstab Akteure einen Konflikt betrachten und i. w. S. auf welchem Maßstab Akteure primär handeln können. So könnte i. w. S. ein globaler Akteur wie die Europäische Union sowohl auf nationaler, regionaler und lokaler Ebene handeln, während ein lokaler Akteur wie ein*e regionale*r Landwirt*in in erster Linie nur auf lokaler Ebene handeln kann.

Zudem können Akteure in zwei Akteursarten unterteilt werden, welche die Art der Betrachtung wiedergeben (vgl. Weber 1980; Reuber 2001; Schimank 2011). So entspricht ein direkter Akteur einer konkreten Person (z. B. Politiker*innen, Einwohner*innen, Umweltschützer*innen), sodass hier ein Sachverhalt oder Konflikt aus der Perspektive einer konkret handelnden Person betrachtet wird. Bei einem indirekten Akteur erfolgt hingegen die Betrachtung fachspezifisch (z. B. wirtschaftlich, gesellschaftlich, ökologisch), 
d.h., dass der Sachverhalt oder Konflikt nicht aus der Perspektive einer konkreten Person erfolgt, sondern ausschließlich aus einer Perspektive, die an eine Fachdisziplin (z. B. Wirtschaft, Ökologie oder Politik) gebunden ist. Anzumerken ist hierbei, dass nur direkte Akteure sinnhaft orientiert Handeln können.

Weiterhin können direkte Akteure in zwei Akteurstypen unterteilt werden (vgl. Scharpf 2000: 95 ff.), den individuellen Akteurstyp (z.B. Einwohner*in) und den komplexen Akteurstyp (z. B. Vertreter*in eines Unternehmens, politische* ${ }^{*}$ Entscheidungsträger ${ }^{*}$ in). Während individuelle Akteure die eigenen Interessen verfolgen, sind komplexe Akteure an die Interessen der jeweiligen Institution (z. B. Unternehmen oder staatliche Einrichtungen) gebunden.

Die Handlungs- und Durchsetzungsfähigkeit der Interessen und Ziele der Akteure hängt oftmals von der Akteursebene und dem Akteurstyps ab. So können lokale Akteure (z. B. Bürgermeister*in) ihre Interessen in einem Konflikt oftmals weniger wahrscheinlich durchsetzen als globale Akteure (z. B. Europäische Union). Bei der Handlungs- und Durchsetzungsfähigkeit kann jedoch nicht nur die Akteursebene sondern auch der Akteurstyp entscheidend sein. So weisen komplexe Akteure (z.B. Vertreter*in eines Unternehmens) oftmals aufgrund von bspw. vorhandenen Netzwerken, finanziellen Ressourcen oder potentieller Entscheidungsgewalt eine andere Handlungsfähigkeit und demnach eine stärkere Durchsetzungsfähigkeit ihrer Interessen und Ziele auf als individuelle Akteure (z. B. Einwohner*in).

Abb. 1 zeigt die idealtypische Unterteilung auf, durch die Akteure genauer definiert werden können.
Anzumerken ist hierbei, dass in der abgeleiteten Klassifikation im Sinne der Argumentations- und Diskursanalyse weder die Argumente der Akteure analysiert noch die genauen Machtverhältnisse, die vorhandenen Ressourcen oder die genaue Fähigkeit der Akteure zu strategischen Entscheidungen dargestellt werden. Die Klassifikation dient daher primär dazu, die vorhandenen Akteure in einem Konflikt zu erfassen und zu strukturieren. Dadurch kann die Klassifikation bei der didaktischen Reduktion Hilfestellung geben, wenn die an einem Konflikt beteiligten Akteure für den Perspektivenwechsel gewählt werden müssen, um den Schüler*innen den Konflikt in seiner Komplexität und Kontroversität darzustellen. Im folgenden Kapitel werden daher die didaktischen Hürden kurz aufgezeigt, die bei der Wahl der Akteure bestehen können.

\subsection{Die Wahl der Akteure: Hürden bei der didaktischen Reduktion}

Aufgrund subjektiver Raumwahrnehmungen der Akteure ist der Geographieunterricht dafür prädestiniert, Schüler*innen mithilfe des Perspektivenwechsels verschiedene Sichtweisen auf einen Konflikt erschließen zu lassen. Hierbei ist die Aufgabe der Lehrkraft bei der Unterrichtskonzeption eine didaktisch quantitative Reduktion (Stoffreduktion) und eine didaktisch qualitative Reduktion (Inhaltsreduktion) durchzuführen. Es bestehen allerdings verschiedene Hürden bei der didaktischen Reduktion. In Bezug auf die quantitative Reduktion unterliegen viele Lehrkräfte der sogenannten Vollständigkeitsfalle, d.h. dem Wunsch, den Inhalt möglichst vollständig zu lehren (vgl. Leh-

\section{Akteure}

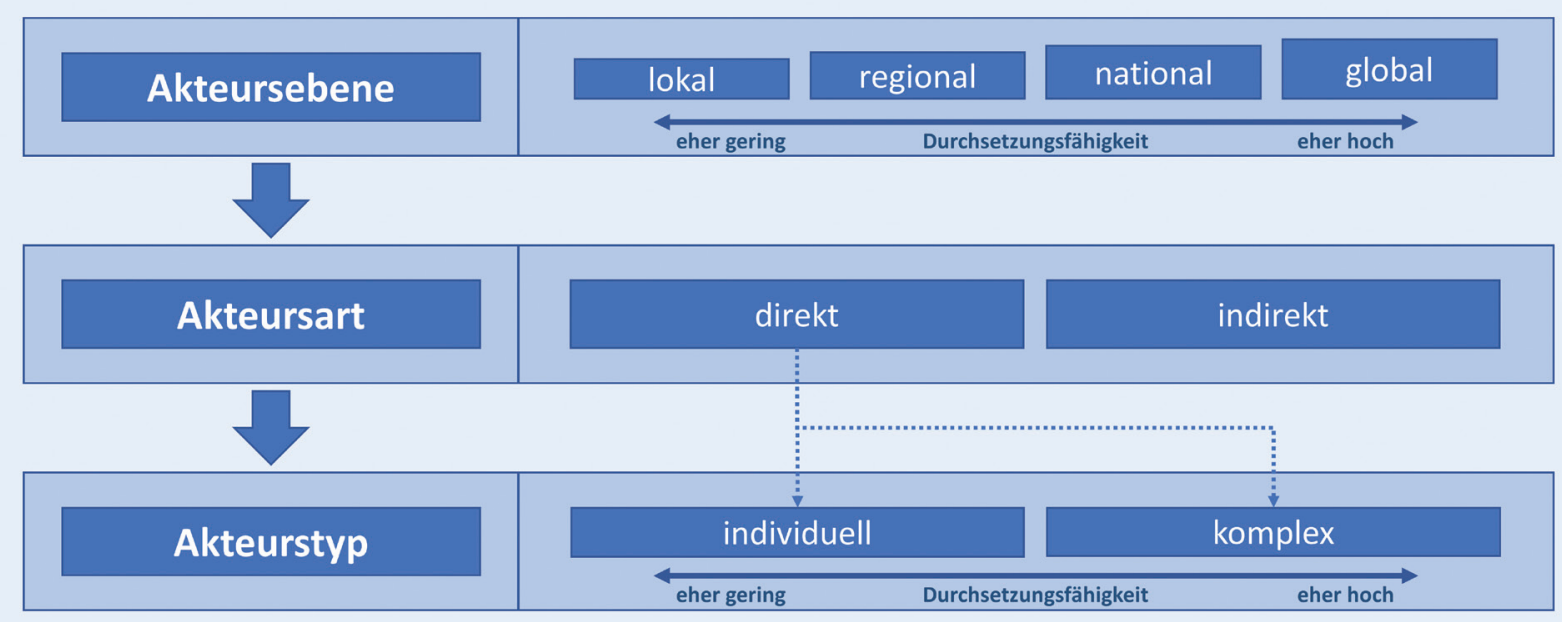

Abb. 1: Dimensionen, durch die Akteure genauer definiert werden können (eigene Darstellung) 
ner 2011: 29 ff.). Das Bestreben, die zu behandelnden Themen möglichst „ohne quantitative Reduktion zu lehren ist verständlich, aber didaktisch nicht akzeptabel“ (Aschersleben 1993: 141). So können auch im Geographieunterricht nicht alle an einem Konflikt beteiligte Akteure dargestellt werden. Die Lehrkraft muss daher bei der Unterrichtskonzeption eine Auswahl treffen, bei der allerdings darauf zu achten ist, dass die in einem Konflikt betroffenen Akteure und ihre Interessen nicht zu einseitig dargestellt werden. So sollte nach den Prinzipien der politischen Bildung (Beutelsbacher Konsens) bei der Unterrichtskonzeption das Kontroversitätsprinzip unbedingt berücksichtigt werden. Dieses besagt: „Was in Wissenschaft und Politik kontrovers ist, muß auch im Unterricht kontrovers erscheinen" (Wehling 1977: 179). So ist es trotz einer quantitativen Reduktion wichtig, verschiedene Standpunkte aufzuzeigen und Alternativen erörtern zu lassen, sodass den Schüler*innen im Unterricht eine freie Meinungsbildung ermöglicht wird. In diesem Zusammenhang kann bei der Darstellung eines Konflikts zudem die Maßstäblichkeit der Sichtweisen (kleiner, mittlerer oder großer Maßstab) berücksichtigt werden. So kann ein Konflikt von den Schüler*innen unterschiedlich wahrgenommen werden, je nachdem, welche Maßstäblichkeit bei der Darstellung gewählt wird. Eine Betrachtung aus lokaler Perspektive kann andere Aspekte eines Konflikts offenlegen als eine Betrachtung aus globaler Perspektive. Daher können mithilfe der Maßstäblichkeit die Interessen der verschiedenen beteiligten Akteure und somit die unterschiedlichen oder gar widersprüchliche Betrachtungsweisen für die Schüler*innen offengelegt (vgl. Rhode-Jüchtern 2011: 9ff.) und die Ambiguitätstoleranz gefördert werden (vgl. Kiehl \& Schnerch 2018: 116).

Bei der qualitativen Reduktion muss die Lehrkraft einerseits das Wesentliche eines Themas erfassen, andererseits das Komplizierte eines Sachverhalts vereinfacht darstellen (vgl. Vogel 1995: 567). Es muss jedoch darauf geachtet werden, dass keine zu starke Vereinfachung erfolgt. Denn wird „Komplexität im Vorraum von Unterricht beseitigt, lässt sich nicht mehr lernen, Komplexität zu beherrschen" (Kell \& Kutscha zit. nach Lehner 2020: 141). Daher besteht bei der Reduktion komplexer Zusammenhänge im Raum die Gefahr, „die kulturellen und wirtschaftlichen Eigenschaften dort lebender Menschen" (Kaminske 2012: 163) zu vereinfachen und Stereotypen zu bilden, weil wegen mangelnder Kenntnisse der Schüler*innen oft Vorstellungsbilder durch „Hilfskonstruktionen wie Klischees, Stereotypen und Vorurteile ergänzt oder ersetzt" werden (Kaminske 2012: 163). Da der Geographieunterricht die Vorstellungen der Schüler*innen prägt, ist es wichtig, dass der Geographieunterricht nicht zur Bildung von Stereotypen beiträgt. So wurde z. B. bereits in verschiedenen Studien aufgezeigt, dass eine einseitige Darstellung Afrikas u.a. auch in Schulbüchern erfolgt und Stereotypen gefördert werden (vgl. Tröger 1993; Arndt 2006). Das hat zur Folge, dass deutsche Schüler*innen Afrika mitunter als einen Kontinent wahrnehmen, der insgesamt als arm und rückständig anzusehen ist (vgl. Schmidt-Wulffen 1997). Daher muss bereits bei der Materialentwicklung darauf geachtet werden, welche Akteure bei der Darstellung eines Sachverhalts oder Konflikts gewählt werden, damit keine Simplifizierung erfolgt und somit auch vermeidbare Stereotypen nicht gefördert werden.

\section{Forschungsmethodisches Vorgehen}

Ziel der Analyse war es, zum einen zu untersuchen, welche Akteure bei Konflikten im Rahmen des Perspektivenwechsels von den Autor*innen gewählt werden, zum anderen inwiefern die gewählten Akteure die Komplexität des thematisierten Konflikts aufzeigen. Für die empirische Analyse wurden 28 Artikel aus deutschsprachigen geographiedidaktischen Praxiszeitschriften gewählt. Die Publikationen mussten insgesamt vier Kriterien erfüllen. In Titel oder Untertitel des Aufsatzes musste mindestens einer der folgenden Begriffe verwendet werden: Perspektive, Perspektiven, Perspektivenwechsel, Perspektivwechsel, Mehrperspektivität, Perspektivismus, Perspektivität, Multiperspektivität, Vielperspektivität, Perspektivität, Perspektivenübernahme, Perspektivübernahme, multiperspektivisch, mehrperspektivisch, vielperspektivisch, Fremdverstehen, interkulturelles Lernen, Interkulturalität, Rollenübernahme und Rollenspiel. Damit wird anhand der Titelwahl wahrscheinlich ersichtlich, dass das Prinzip der Multiperspektivität für die Autor*innen in der didaktischen Umsetzung im Fokus steht. Aufbauend darauf konnten im weiteren Schritt die Akteure im Rahmen des Perspektivenwechsels analysiert werden. Aufsätze von Autor*innen, die den Begriff Perspektive als $\mathrm{Zu}$ kunftsaussicht verwenden und sich dabei nicht mit verschiedenen Standpunkten auseinandersetzen, wurden nicht zur Analyse herangezogen. Zweites Kriterium war das Vorhandensein von entsprechenden didaktischen Ausführungen. Diese konnten Aufschluss darüber geben, weshalb die Autor*innen bestimmte Akteure beim Perspektivenwechsel gewählt haben und welche Ziele sie damit verfolgten. Drittes Kriterium bei der Auswahl der Aufsätze war die materialgestützte Ausarbeitung der Unterrichtseinheit, damit zum einen analysiert werden konnte, ob die Akteure real oder fiktiv sind, zum anderen wie die Akteure didaktisch ausgearbeitet und dargestellt wurden. Die 
Ergebnisse der Analyse der Methoden und Medien war allerdings nicht aussagekräftig genug, sodass sie nicht weiter in den Ergebnissen dargestellt werden. Aufsätze, die nur für den Themeneinstieg Material zur Verfügung stellen und z. B. eine Projektarbeit als Ziel verfolgen, wurden nicht zur Analyse hinzugenommen. Viertes Kriterium war das Vorhandensein von Aufgabenstellungen, da diese konkrete Anweisungen geben können, wie sich die Schüler*innen beim Perspektivenwechsel mit den Akteuren auseinandersetzen sollen. Autor*innen, die den gleichen Artikel in zwei Publikationsorganen veröffentlicht haben, wurden für die Analyse nur einmalig berücksichtigt. Hierbei wurde der Artikel zur Analyse gewählt, der zuerst veröffentlicht wurde. Eine Jahresbeschränkung der Publikationen erfolgte nicht. Insgesamt erfüllten 28 Aufsätze diese Kriterien. Sie wurden zwischen 1996 und 2017 in den geographiedidaktischen Zeitschriften Geographie aktuell \& Schule, GW-Unterricht, geographie heute und Praxis Geographie veröffentlicht. Die Analyse der Akteure in den geographiedidaktischen Artikeln erfolgte nach der inhaltlich strukturierenden qualitativen Inhaltsanalyse von Mayring (vgl. 2015) und Kuckartz (vgl. 2018). Die vorgenommene Bildung der Analysekategorien erfolgte induktiv-deduktiv.

Vor der eigentlichen Akteursanalyse wurde zunächst erfasst, ob Akteure im Rahmen eines Konflikts dargestellt wurden. Unter einem Konflikt wurden dabei, wie bereits in der Einleitung dargelegt wurde, Zielvorstellungen von Akteuren verstanden, die nicht miteinander vereinbar sind (vgl. Bonacker 2009: 181). Hierbei wurde unterschieden, ob der Konflikt explizit oder implizit war. Ein expliziter Konflikt bedeutete, dass die Autor*innen in den didaktischen Ausführungen den Konflikt direkt benannt haben, wie im folgenden Beispiel: „Der Stadtteil, in dem es zu dem Konflikt kommt" (Hofmann et al. 2016: 35). Bei impliziten Konflikten hingegen wurden die verschiedenen Meinungen der Akteure von den Autor*innen nicht konkret als unvereinbare Zielvorstellungen dargelegt, wie im folgenden Beispiel: „[...] welche die ökologischen und ökonomischen Komponenten des Themas den Schülerinnen und Schülern aufzeigt. Sie sollen versuchen, sachlich zu argumentieren und die vielfältigen Betrachtungsweisen gegeneinander abzuwägen" (Riediger 1997: 26). Unterrichtsbeiträge, die weder einem expliziten noch impliziten Konflikt zugeordnet werden konnten, wurden in der Kategorie kein Konflikt erfasst. Danach wurde untersucht, ob die Darstellung des Konflikts oder Sachverhalts an einem konkreten Raumbeispiel erfolgte. Dabei wurde die Maßstabsebene (lokal, regional, national, global) bestimmt, auf der das Raumbeispiel dargestellt wurde. Anschließend wurden die einzelnen Akteure gemäß der definierten Dimensionen (siehe Abb. 1) analysiert. Im folgenden
Abschnitt werden nun die Schritte der Akteursanalyse genauer beschrieben.

Jeder Akteur wurde zuerst der Akteursebene zugewiesen, um bestimmen zu können, ob er lokal, regional, national oder global agiert, denn nach RhodeJüchtern (vgl. 2011: 9 ff.) sollte bei der Darstellung des Konflikts auch die Maßstäblichkeit von Perspektive und Akteur berücksichtigt werden. Anschließend wurde die Zuweisung mit der vorherigen Maßstabszuordnung des Raumbeispiels verglichen. Dabei wurde u. a. herausgearbeitet, ob bei einem nationalen Beispiel wie den Malediven (vgl. Dolić \& Wilhelmi 2011) nur nationale oder auch andere Akteur gewählt wurden. So kann die Wahl des Maßstabs u. a. aufzeigen, welche Interessen der Akteure berücksichtigt werden. Die Autoren haben z. B. bei dem Raumbeispiel Malediven (vgl. Dolić \& Wilhelmi 2011) neben dem nationalen Akteur wie Mohammed Nasheed (Staatspräsident der Malediven) auch einen lokalen Akteur wie Bill Allison (Gastarbeiter) ausgesucht. Anschließend wurde erfasst, welche Akteursart (direkt oder indirekt) für den Perspektivenwechsel in den analysierten Unterrichtsvorschlägen gewählt wurde. So haben sich z. B. die Autoren Dolić \& Wilhelmi (vgl. 2011) in ihrem Unterrichtsvorschlag für direkte Akteure entschieden, da Mohammed Nasheed und Bill Allison konkrete Personen sind, die handeln. Die direkten Akteure wurden zudem dahingehend analysiert, ob es sich bei der Darstellung um real existierende Akteure oder fiktive direkte Akteure handelt. So handelt es sich bei den direkten Akteuren Mohammed Nasheed (Staatspräsident der Malediven) und Bill Allison (Gastarbeiter) um reale direkte Akteure (vgl. Dolić \& Wilhelmi 2011: 52). Weiterhin wurde erfasst, welcher direkte Akteurstyp (individuell oder komplex) gewählt wurde. So handelt es sich bei dem direkten Akteur Mohammed Nasheed um einen komplexen Akteur, der die Interessen der Bevölkerung vertritt und bei Bill Allison um einen individuellen Akteur, der ausschließlich seine eigenen Interessen verfolgt (vgl. Dolić \& Wilhelmi 2011: 52). Darüber hinaus wurde auch erfasst, ob die Schüler*innen in den Unterrichtsvorschlägen aufgefordert werden, die Handlungs- sowie Durchsetzungsfähigkeit der Akteure zu berücksichtigen. Es wurde hierfür betrachtet, ob die Aufgabenstellungen auf die Akteursebene (lokal, regional, national, global) und/oder auf den Akteurstyp (individuell, komplex) oder in Anlehnung an Reuber (vgl. 2001) auf autoritative Ressourcen (z. B. Netzwerke, politische Position im Entscheidungssystem), allokative Ressourcen (z. B. finanzielle Potentiale, Grund und Boden) und / oder auf individuelle Komponenten (z. B. persönliches Charisma, Durchsetzungsstärke) Bezug nehmen. Anzumerken ist hierbei, dass die Schüler*innen in keinem geographiedidaktischen Artikel die Handlungs- 
oder Durchsetzungsfähigkeit der Akteure in einem Konflikt berücksichtigen mussten.

\section{Ergebnisse}

Im Folgenden werden ausgewählte Ergebnisse der Analyse präsentiert, um anschließend diskutieren zu können, inwiefern die gewählten Akteure die Komplexität der thematisierten Konflikte aufzeigen. Dabei wird aufgezeigt, ob in den Unterrichtskonzepten ein Konflikt dargestellt wurde. Anschließend werden die Maßstabsebene der Raumbeispiele und die Akteursebene vorgestellt, für die sich die Autor*innen entschieden haben. Zuletzt wird die Wahl der Akteursarten und Akteurstypen dargestellt.

\subsection{Akteure im Kontext eines Konflikts}

Insgesamt ist festzustellen, dass die Autor*innen in elf der 28 analysierten Artikel einen expliziten Konflikt in der Unterrichtskonzeption darlegen, wie in folgendem Beispiel: „Erklären Sie auf der Basis, wie verschiedene Akteure/Gruppen den Raum wahrnehmen, die sich abzeichnenden Konflikte" (Coen et al. 2017: 33). In neun der analysierten 28 Artikel werden implizite Konflikte in Form unterschiedlicher Positionen aufgezeigt ohne den Sachverhalt konkret als Konflikt zu benennen, wie folgendes Beispiel aufzeigt: „Die unterschiedlichen Positionen, die beliebig erweitert oder ausgetauscht werden können, konfrontieren die Schülerinnen und Schüler [...] mit den positiven und negativen Auswirkungen des Tourismus" (Hemmer 1996: 19). Dennoch ist erkennbar, dass unterschiedliche Zielvorstellungen vorliegen, die u.U. unvereinbar sein könnten. Acht analysierte Artikel weisen keinen Konflikt auf. Dabei stehen in sechs der acht Artikel die verschiedenen Raumwahrnehmungen der Akteure im Vordergrund. Die Schüler*innen sollen hierbei den Raum multiperspektivisch erfassen, indem sie z.B. die Perspektiven von verschiedenen Anwohner*innen wie Familien, Paare, Berufstätige, Kinder, Menschen mit Behinderung, Senior*innen, Obdachlose oder Migrant*innen (vgl. Hepp 2017: 21) einnehmen. Hierbei sollen die Schüler*innen darauf sensibilisiert werden, dass der Raum aus verschiedenen Perspektiven wahrgenommen werden kann. Die Autor*innen verfolgen nicht vorrangig das Ziel, unvereinbare Zielvorstellungen der Akteure im Sinne der Definition eines Konflikts darzustellen. In zwei weiteren Unterrichtskonzeptionen steht die Förderung der Empathiefähigkeit der Schüler*innen im Vordergrund. So sollen sie sich ,in die Lebenslage eines Menschen, in diesem Fallbeispiel eines minderjährigen syrischen Flüchtlings" (Fridrich 2017: 28) ver- setzen und sein Lebensgefühl bewerten. Somit steht die Wahrnehmung des syrischen Flüchtlings im Mittelpunkt, sodass „ein kritisches Überdenken des eigenen Standpunkts" (Fridrich 2017: 28) erzielt werden kann. Insgesamt ist festzustellen, dass die Autor*innen Akteure im Rahmen des Perspektivenwechsels primär verwenden, um einen Konflikt oder strittigen Sachverhalt darzustellen.

\subsection{Die Wahl der Maßstabsebene der Raumbei- spiele und der Akteursebene}

In 24 der 28 analysierten Artikel verwenden die Autor*innen konkrete und reale Raumbeispiele, wie in diesem Beispiel: „Die Gemeinde Nendaz [...] hat ca. 5500 Einwohner, von denen ein Großteil vom Tourismus lebt" (Mentz 2002: 22). Bei zwei analysierten Artikeln soll der eigene Wohnort analysiert werden. Bei zwei weiteren Beispielen ist der Raum nicht näher bestimmt, wird aber als idealisierter Realraum vorgestellt: „Der Stadtteil, in dem es zu dem Konflikt kommt, ist ein bürgerlich geprägtes Wohnviertel [...]" (Hofmann et al. 2016: 35). Es ist daher deutlich zu erkennen, dass vorrangig konkrete und reale Raumbeispiele von den Autor*innen als Grundlage für den Perspektivenwechsel verwendet werden und kaum Raumbeispiele, die geographisch nicht konkret zu lokalisieren sind.

Insgesamt sind 14 der erfassten Raumbeispiele dem lokalen Maßstab, neun dem nationalen, zwei dem regionalen und drei dem globalen Maßstab zuzuordnen. Somit werden von den Autor*innen in 50\% der analysierten Artikel lokale Raumbeispiele gewählt.

Neben dem Maßstab des Raumbeispiels ist die Akteursebene von Interesse. Festzustellen ist, dass alle Autor*innen mindestens einen Akteur auswählen, dessen Maßstabsebene der des Raumbeispiels entspricht. Dabei beschränken sich die Autor*innen in 14 der 28 analysierten Artikel nur auf eine Akteursebene. Auffällig ist dabei, dass die Autor*innen in elf dieser 14 Artikel lokale Raumbeispiele auswählen und dementsprechend nur lokale Akteure und somit die lokale Akteursebene für den Perspektivenwechsel wählen. So ist z. B. das Raumbeispiel Nendaz (vgl. Mentz 2002: 23 ff.) der lokalen Maßstabsebene zuzuordnen, ebenso wie die vier ausgewählten Akteure Einwohner von Nendaz, der Verkehrsverein von Nendaz, die Naturschützer sowie die örtlichen Politiker. Dabei verfolgt Mentz (2002: 22) das Ziel, eine "Annäherung an den Konflikt Umwelt-Tourismus" zu erreichen. Es könnten jedoch bei dem Beispiel Nendaz (vgl. Mentz 2002: 22) die Interessenskonflikte und widersprüchlichen Zielsetzungen der verschiedenen Akteure nicht nur auf lokaler, sondern z.B. auch auf nationaler Ebene dargestellt werden, wenn neben den lokalen auch nationale Akteure herangezo- 
gen würden, wie der*die Wirtschaftsminister*in oder der*die Minister*in für Umwelt und Naturschutz. Zusammenfassend wählen die Autor*innen in $14 \mathrm{Ar}$ tikeln eine Akteursebene, in elf der analysierten 28 geographiedidaktischen Artikeln zwei Akteursebenen und in drei der analysierten Artikel drei Akteursebenen für die Darstellung eines Sachverhalts oder Konflikts aus. In keinem der analysierten Artikel werden Akteure gewählt, die insgesamt alle vier Akteursebenen vertreten.

\subsection{Die Wahl der Akteursart und des Akteurstyps}

Die Erarbeitung eines Sachverhalts oder Konflikts kann aus der Betrachtung von direkten als auch indirekten Akteuren erfolgen. Während bei direkten Akteuren die Sichtweise an konkrete Personen gebunden ist, handelt es sich bei indirekten Akteuren um eine fachspezifische Betrachtung, die an keine konkrete Person gebunden ist. So soll z. B. der Massentourismus in Benidorm mit indirekten Akteuren multiperspektivisch betrachtet werden, indem dieser ökologisch, sozial und ökonomisch analysiert wird (vgl. Rempfler \& Landtwing Blaser 2016: $27 \mathrm{ff}$.). Dagegen soll z. B. der Tourismus bei dem Beispiel Wolkenstein mit direkten Akteuren wie dem Landwirt, Pensionswirtin Fran Meier, Holzschnitzer Urs oder Umweltschützerin Frau Poldinger (vgl. Hemmer 1996: $20 \mathrm{f}$.) multiperspektivisch untersucht werden. Wird betrachtet, welche generelle Akteursart (direkt, indirekt) für die Darstellung des Konflikts von den Autor*innen gewählt wird, ist auffällig, dass sich fast vierfach so viele Autor*innen der geographiedidaktischen Artikel $(\mathrm{N}=22)$ für eine Betrachtung entscheiden, die an konkrete Personen gebunden ist (direkte Akteure). Lediglich sechs Autor*innen entscheiden sich dagegen für eine fachspezifische Betrachtung des Konflikts (indirekte Akteure). Wird die Darstellung der direkten Akteure $(\mathrm{N}=22)$ genauer betrachtet, ist festzustellen, dass sich die Autor*innen in acht Artikeln dazu entscheiden, handelnde Personen zu wählen, die in der Realität existieren, während in zwölf Artikeln fiktiv handelnde Personen dargestellt werden. Bei zwei analysierten Aufsätzen konnte nicht nachvollzogen werden, ob die von den Autor*innen gewählten Akteure real oder fiktiv sind. Insgesamt ist festzustellen, dass die Autor*innen entweder nur fiktive oder nur reale direkte Akteure wählen. Eine Mischform wird von den Autor*innen nicht vorgenommen.

Neben der Akteursebene kann die Einteilung der Akteure in Akteurstypen (individuell, komplex) Auskunft darüber geben, welche Interessen dargestellt werden und welche davon mit einer höheren Handlungs- und Durchsetzungsfähigkeit verbunden sind. So können komplexe Akteure aufgrund von vorhan- denen Organisationsstrukturen ihre Interessen oftmals stärker durchsetzen als individuelle Akteure. In den analysierten Aufsätzen sind insgesamt 129 direkte Akteure dargestellt von denen 60 individuelle und 55 komplexe Akteure sind. 14 Akteure konnten nicht zugeordnet werden, da nicht ersichtlich war, ob der Akteur eine Organisation vertritt und somit ein komplexer Akteur ist oder als individueller Akteur auftritt wie die Umweltschützerin Frau Poldinger (vgl. Hemmer 1996: 20). Insgesamt werden in $50 \%$ der analysierten Aufsätze sowohl individuelle als auch komplexe direkte Akteure für den Perspektivenwechsel gewählt, wie Senhor Lobo Carniceiro, Direktor eines Zellulosekonzerns oder Ernesto, der Bauer (vgl. Schramke \& Uhlenwinkel 2004: 30). Somit zeigt sich, dass sich einige Autor*innen für eine Darstellung entschieden haben, die die Komplexität eines Sachverhalts oder Konflikts mithilfe verschiedener Akteurstypen aufzeigt. Allerdings müssen die Schüler*innen, wie bereits in Kapitel 2 erwähnt, in keinem der analysierten Aufsätze die Handlungs- oder Durchsetzungsfähigkeit der Akteure hinterfragen oder erläutern.

\section{Diskussion}

Ein Ziel der Analyse war es, zu untersuchen, welche Akteure in den geographiedidaktischen Artikeln von den Autor*innen im Rahmen des Perspektivenwechsels ausgewählt wurden, um einen Sachverhalt oder Konflikt im Unterrichtsmaterial darzustellen. Das zweite Ziel war zu erfassen inwiefern die gewählten Akteure die Komplexität des thematisierten Konflikts aufzeigen. Hierfür wurde eine Klassifikation entworfen, die die Dimensionen Akteursebene, Akteursart und Akteurstyp umfasst (siehe Abb. 1). Anschließend wurden die Akteure in den analysierten Artikeln den einzelnen Dimensionen zugeordnet. In diesem $\mathrm{Zu}$ sammenhang wurde auch untersucht, welcher Maßstabsebene die ausgewählten Raumbeispiele zugewiesen werden konnten.

In $50 \%$ der analysierten Artikel werden von den Autor*innen lokale Raumbeispiele gewählt. Das könnte daran liegen, dass es möglicherweise für Schüler*innen einfacher sein kann, ein auf einen kleinräumigen Maßstab angesiedeltes Beispiel für den Perspektivenwechsel zu nutzen, weil dies schneller zu erfassen ist. Wie erwartet stimmt die Maßstabsebene des Raumbeispiels auch immer mit der Maßstabsebene der Akteure überein. Wenn z. B. ein nationales Raumbeispiel ausgesucht wurde, wurden auch national handelnde Akteure gewählt (vgl. Coen et al. 2017). Dabei kann die Wahl der Akteursebenen sich darauf auswirken, wie die Schüler*innen die Kontroversität eines Konflikts wahrnehmen (vgl. Nehrdich 2011: 
$15 \mathrm{ff}$.; Rhode-Jüchtern 2011: 9). Allerdings wird in 14 der 28 analysierten Artikel nur eine Akteursebene gewählt, um den Konflikt zu veranschaulichen. Dabei ist hervorzuheben, dass die Autor*innen von elf dieser 14 Artikel lokale Raumbeispiele gewählt und demensprechend nur lokal handelnde Akteure ausgesucht haben. Insgesamt werden somit in 39\% der analysierten Artikel lokale Raumbeispiele und lokale Akteure für die Darstellung eines Konflikts ausgesucht. Weisen Raumbeispiel und Akteure denselben Maßstab auf, ermöglicht das zwar grundsätzlich eine vertiefte Darstellung des entsprechenden Konflikts oder Sachverhalts. Zeitgleich besteht jedoch das Risiko, einen Konflikt nicht in seinem tatsächlichen Spektrum aufzuzeigen (vgl. Wehling 1977: 179). Mit einem Maßstabswechsel können allerdings Widersprüche in der Wahrnehmung des Problems deutlicher dargestellt und die Schüler*innen für die räumliche Gebundenheit der Akteursperspektiven sensibilisiert werden (vgl. Rhode-Jüchtern 2011: 9). Mithilfe der verschiedenen Akteursebenen können den Schüler*innen die Komplexität eines Konflikts und die realen Schwierigkeiten einer Lösungsumsetzung aufgezeigt werden, da Akteure verschiedener Akteursebenen unterschiedliche Facetten eines Sachverhalts oder Konflikts aufgreifen. Zudem könnten anhand der unterschiedlichen Akteursebenen Diskrepanzen oder Ähnlichkeiten der verschiedenen verfolgten Interessen, die dabei vertretenen Werte und die Durchsetzungsfähigkeit der Ziele klarer herausgestellt werden. Wie bereits ausgeführt (siehe Kap. 3.1.) könnten z. B. beim Raumbeispiel Nendaz (vgl. Mentz 2002: 23 ff.) Interessenskonflikte und widersprüchliche Zielsetzungen bei dem Umwelt-Tourismus-Konflikt nicht nur auf lokaler, sondern auch auf nationaler Ebene dargestellt werden. Zudem kann ein Wechsel der Akteursebene aufzeigen, dass getroffene Entscheidungen auf einer Maßstabsebene Auswirkungen auf andere Ebenen haben können, ohne dass auf dieser eine Verhandlung stattgefunden hat (vgl. Lambert \& Morgan 2010). Die Analyse zeigt, dass die Potentiale der unterschiedlichen Akteursebenen im Sinne eines räumlichen Maßstabwechsels im Rahmen des Perspektivenwechsels in den analysierten Unterrichtsvorschlägen nicht gänzlich genutzt werden.

Bei der Analyse der Akteursart (direkt oder indirekt) ist festzustellen, dass die Mehrheit der Autor*innen die Sichtweise von konkreten Personen wählen $(N=22)$ und sich weniger für eine fachspezifische Betrachtung des Sachverhalts oder Konflikts entscheiden $(\mathrm{N}=6)$. Möglicherweise verfolgen sie damit das Ziel, anhand von direkten Akteuren Interessen und Einstellungen zu verdeutlichen, sodass die Meinungsverschiedenheit von den Schüler*innen verstanden werden können. Zudem können schwer zugängliche
Themen für Schüler*innen eingängiger sein, wenn Ereignisse an direkte Akteure gebunden sind, wie die Lebensstationen des Flüchtlings Yusufs aus Syrien (vgl. Fridrich 2017: 28 ff.). Hierbei steht die Förderung der Empathiefähigkeit im Vordergrund, mit der Emotionalität als Basis für den Erkenntnisprozess (vgl. Kruckemeyer 1995: 114). In diesem Zusammenhang werden allerdings andere Perspektiven und Akteure, wie Anwohner*innen oder Politiker*innen, die zum Verständnis von Migration beitragen könnten, nicht in das Unterrichtsmaterial integriert.

Auffällig ist zudem, dass sich die Autor*innen in zwölf Artikeln dafür entscheiden, den Sachverhalt oder Konflikt mithilfe von fiktiven direkten Akteuren darzustellen. Lediglich in acht Artikeln veranschaulichen die Autor*innen den Sachverhalt oder Konflikt mithilfe von realen direkten Akteuren, die auch in der Realität existieren. Allerdings vermischen die Autor*innen niemals fiktive und reale Akteure. Mithilfe von fiktiven direkten Akteuren kann ein Konflikt idealtypisch aufbereitet werden, indem die Komplexität eines Sachverhalts oder Konflikts didaktisch reduziert und damit an das Leistungsniveau der Schüler*innen angepasst wird. Allerdings können gerade fiktive Darstellungen von direkten Akteuren dazu führen, dass Stereotypen hinsichtlich ihrer Wertvorstellungen, Ziele und Interessen verstärkt werden, wenn z. B. fiktive direkte Akteure - wie die Indianerin, Pressesprecher eines industriellen Großprojekts, Manager eines internationalen Fleischkonzerns (vgl. Endreß 2003: 36) oder der „Bauunternehmer: riecht das große Geld durch Bauaufträge, kann eigenes Land verkaufen, stellte schöne Projekte vor, kann seine Ferienwohnung vermieten“ (Reuschenbach 2007: 16) - verwendet werden. Wenn solch eine Simplifizierung der Akteure erfolgt, werden deren individuelle Eigenschaften zugunsten einer idealtypischen Darstellung beseitigt. Schüler*innen könnten dadurch ihre Vorstellungbilder um im Unterricht vermittelte Stereotypen oder Klischees ergänzen (vgl. Kaminske 2012: 163). Wenn dagegen mit realen Akteuren gearbeitet wird, ist solch eine Simplifizierung oder Stereotypisierung bei der Darstellung kaum möglich, da Aussagen von realen Akteuren verwendet werden, die referenziert werden müssen. Daher sollte bei der Darstellung eines Konflikts mithilfe von fiktiven Akteuren darauf geachtet werden keine typischen Rollen auszuarbeiten, die den Konflikt unnötig simplifizieren. Zudem sollten die Schüler*innen darauf hingewiesen werden, wenn fiktive Akteure im Unterrichtsmaterial verwendet werden und der Konflikt daher eher einen exemplarischen Charakter aufweisen soll. Im Sinne der Ambiguitätstoleranz kann es demnach förderlicher sein, wenn die Schüler*innen lernen, dass Meinungen von realen direkten Akteuren Widersprüchlichkeiten in der Wahrnehmung eines 
Konflikts aufweisen. Sie lernen somit verschiedene Positionen anzuerkennen und vor allem auch auszuhalten (vgl. Frenkel-Brunswik 1949: 108 ff.). So kann mithilfe der Ambiguitätstoleranz die Mündigkeit der Schüler*innen im Geographieunterricht gefördert werden, was eine zentrale Demokratiekompetenz darstellt (vgl. Kiehl \& Schnerch 2018: 116).

In 24 der 28 analysierten Artikel werden reale Raumbeispiele gewählt, weswegen sich die Frage stellt, ob real existierende Konflikte nicht auch mit realen Akteuren dargestellt werden sollten, um beim Perspektivenwechsel eine Auseinandersetzung mit den realen Beweggründen der direkten Akteure zu erzielen. So sollten die Schüler*innen bei der Auseinandersetzung mit verschiedenen Perspektiven lernen, die verschiedenen Sichtweisen zu dekonstruieren (vgl. Duncker 2005: 9 ff.). Daher sollten die Schüler*innen real existierende Konflikte möglichst aus realen Perspektiven heraus analysieren, verstehen und anschließend reflektieren. Wenn die direkten Akteure analysiert werden, muss anschließend auch herausgestellt werden, für welchen Akteurstyp sich die Autor*innen bei der Darstellung entscheiden. Es ist auffällig, dass in sieben der 28 analysierten Artikel für die Darstellung des Sachverhalts oder Konflikts entweder nur individuelle Akteurstypen oder nur komplexe Akteurstypen gewählt werden. So wählen z. B. Franz \& Trebbin (vgl. 2012: 28) insgesamt vier Akteure für die Darstellung des Sachverhalts aus, um die zentrale Frage beantworten zu können „ob und wem Supermärkte in Indien Vorteile bringen " (Franz \& Trebbin 2012: 26). Dabei werden für die Darstellung direkte Akteure gewählt wie Vinod Shetty (Vertreter der Nichtregierungsorganisation India FDI Watch), N.K. Shukla (National joint secretary of All India Kisan Sabha) oder Michael Wiedmann (Head of Corporate Relations der METRP GROUP), die ausschließlich komplexen Akteurstypen entsprechen. In $50 \%$ der analysierten Artikel wählen die Autor*innen sowohl individuelle als auch komplexe Akteurstypen, wie bei dem Beispiel Eukalyptus in Portugal (vgl. Schramke \& Uhlenwinkel 2004: 30). So müssen sich die verfolgten ökologischen Ziele „mit den ökonomischen und sozialen Interessen der Bevölkerung in Einklang bringen lassen, Interessen, die in sich schon widersprüchlich sein können" (Schramke \& Uhlenwinkel 2004: 28). Um die widersprüchlichen Interessen darzulegen, werden sowohl individuelle Akteurstypen wie Maria, die alte Frau als auch komplexe Akteurstypen wie Senhor Lobo Carniceiro, Direktor eines Zellulosekonzerns gewählt (vgl. Schramke \& Uhlenwinkel 2004: 30). Wenn verschiedene Akteurstypen gewählt werden, kann den Schüler*innen nicht nur aufgezeigt werden, dass Akteure unterschiedliche oder gar widersprüchliche Interessen aufweisen, sondern auch, dass die Akteu- re verschiedene Durchsetzungsfähigkeiten bzgl. ihrer Interessen haben (vgl. Field \& Higley 1983). So können bspw. individuelle Akteure (z. B. Maria, die alte Frau) ihre Interessen und Ziele gegenüber komplexen Akteuren (z. B. Senhor Lobo Carniceiro, Direktor eines Zellulosekonzerns) aufgrund vorliegender Machtasymmetrien oftmals nicht durchsetzen.

Grundsätzlich müssen bei der Erschließung eines raumbezogenen Konflikts nicht beide Akteurstypen ausgesucht werden, da abhängig von der übergeordneten Fragestellung unterschiedliche Aspekte eines Konflikts beleuchtet werden können. Es sollte Schüler*innen dabei aber bewusst sein, welcher Akteurstyp im Rahmen des Perspektivenwechsels ausgewählt wurde. So verfolgen individuelle Akteure eigene Interessen, komplexe Akteure die Interessen einer Institution. Entsprechend sind Beweggründe, Ziele, Handlungs- und Durchsetzungsfähigkeit der Akteurstypen unterschiedlich. Daher sollte die Erfassung der Interessen und Handlungs- und Durchsetzungsfähigkeiten der Akteure in Aufgabenstellungen angeleitet werden. Auffällig ist allerdings, dass die Autor*innen in den Aufgabenstellungen nie explizit auf die unterschiedlichen Akteurstypen und damit auch nicht auf die unterschiedlichen Handlungs- und Durchsetzungsfähigkeiten der direkten Akteure eingehen. Wenn die Autor*innen in ihrem ausgearbeiteten Material die Akteurstypen nicht darlegen, können die Schüler*innen Interessen und Umsetzbarkeit von Lösungsansätzen nicht ausreichend bewerten. Allerdings sollen Schüler*innen im Rahmen der Beurteilungsund Bewertungskompetenz „die unterschiedlichen Interessen von Akteuren aufdecken, eine Situation aus der Sichtweise verschiedener Betroffener betrachten und so auch die Fähigkeit zum Perspektivenwechsel einüben" (DGfG 2017: 23). Somit ist die Analyse des Akteurstyps relevant, damit Schüler*innen eine fundierte Beurteilung und Bewertung im Rahmen eines Perspektivenwechsels vornehmen können.

\section{$6 \quad$ Fazit}

Nach Dunker (2005: 18) fängt Perspektivenwechsel dort an, „wo in Gesprächen Standpunkte voneinander geschieden werden, wo Verständigung gesucht wird, Einfühlungsvermögen gefragt ist und Meinungsverschiedenheiten ausgehalten werden müssen." Gerade im Geographieunterricht werden häufig kontrovers diskutierte Fragestellungen aufgegriffen, „welche auf der Grundlage unterschiedlicher Weltbilder, Interessen der Akteure sowie derer Werte und Normen beantwortet werden" (Budke et al. 2015: 276). Die Analyse der geographiedidaktischen Artikel zeigt auf, dass die Autor*innen im Rahmen 
des Perspektivenwechsels zwar Akteure wählen, die die Kontroversität eines Konflikts oder Sachverhalts darstellen, allerdings werden die Interessen dieser Akteure vorrangig auf nur einer Maßstabsebene aufgezeigt. Es könnte daher für einige Themen zielführender sein, wenn Akteure aus verschiedenen Maßstäben gewählt würden, um die Komplexität eines Sachverhalts oder Konflikts und die widersprüchlichen Sichtweisen der Akteure vertieft darzustellen, um so eine offene Argumentation zu fördern (vgl. Budke et al. 2015: 276). Darüber hinaus soll den Schüler*innen in der Geographie „möglichst viel Authentisches von der Welt und dem Handeln der Menschen in dieser Welt" dargestellt und vermittelt werden (RhodeJüchtern 2004: 57). Ergebnisse der durchgeführten Analyse der geographiedidaktischen Artikel zeigen jedoch auf, dass die Autor*innen überwiegend mit fiktiven Akteuren arbeiten. Das Potential der authentischen Darstellung des Sachverhalts oder Konflikts wird somit nicht gänzlich ausgeschöpft (vgl. Lütje \& Budke 2021). In diesem Zusammenhang sollten neben fiktiven Akteuren auch reale Akteure im Material dargestellt werden, damit einerseits keine Stereotypen und Klischees gefördert werden (vgl. Tröger 1993; Arndt 2006), andererseits die Schüler*innen anhand von realen Sichtweisen lernen, Ambiguitäten zu tolerieren (vgl. Dunker 2005:14). Eine weitere Aufgabe des Geographieunterrichts ist es, den Schüler*innen strukturierende Hilfen „bei der Durchmusterung der Umwelt zu geben, die zwar zur Vereinfachung führen, jedoch nicht zur Verzerrung" (Kaminske 2003: 135). Allerdings müssen die Schüler*innen in keinem der analysierten Artikel die Akteure untersuchen. Damit die Schüler*innen Konflikte und Sachverhalte erschließen können, könnte es demnach hilfreich sein, wenn sie im Geographieunterricht lernen, Akteure und Akteurskonstellationen (vgl. Maynatz \& Scharpf 1995: 49 ff.) in einfacher Form zu analysieren, um somit eine Strukturierung zu erzielen. Durch gezielte Aufgabenstellungen könnten Schüler*innen bspw. angeleitet werden, Akteure bzgl. Akteursebene, Akteursart und Akteurstyp zu klassifizieren und zu erfassen, um so auch komplexe Sachverhalte und Konflikte erschließen, beurteilen und bewerten zu können. Denn ein weiteres Ziel des Geographieunterrichts ist es, Schüler*innen zu mündigen Bürger*innen zu erziehen, die verschiedene Sichtweisen anerkennen, dekonstruieren und verstehen (vgl. Rhode-Jüchtern 2015: 273), um letztlich eine fundierte eigene Meinung bilden zu können. Somit könnten die vorgestellten Dimensionen der Akteure (siehe Abb. 1) sowohl bei der Konzeption des Unterrichtsmaterials als auch bei der Analyse der agierenden Akteure Hilfestellung geben, um den Perspektivenwechsel der Schüler*innen gezielt zu fördern.

\section{Übersicht der untersuchten geographie- didaktischen Artikel}

Bette, J. (2014): Der Raubbau am Regenwald Amazoniens. Eine mehrperspektivische und systemorientierte Raumanalyse. In: Praxis Geographie 3. S. 36-41.

Coen, A., K. W. Hoffmann \& H. Wenz (2017): Rauchen für Malawi. Mit dem Basiskonzept der vier Raumperspektiven einen komplexen Problemzusammenhang entschlüsseln. In: Praxis Geographie 4. S. 28-33.

Dolić, Š. \& V. Wilhelmi (2011): (T)raum Malediven - eine vielperspektivische Raumanalyse. Praxis Geographie 7-8. S. 46-52.

Endreß, K. (2003): Regenwaldkonferenz. Ein Rollenspiel. In: geographie heute 210. S. 34-37.

Fietz, S. (2008): (Un)-Sicherheiten in New York City. Perspektivenwechsel - kritisch denken und differenziert urteilen. In: Praxis Geographie 12. S. 12-17.

Franz, M. \& A. Trebbin (2012): Supermärkte in Indien als Entwicklungschance. Eine Frage der Perspektive. In: Praxis Geographie 9. S. 24-28.

Fridrich, C. (2017): Perspektivenwechsel mit Lebensliniendiagramm und Szenariotechnik - subjektzentrierte $\mathrm{Zu}$ gänge und leistungsdifferenzierte Umsetzungsvarianten. In: GW-Unterricht 145. S. 28-37.

Haversath, J-B. \& S. Hof (2012): Wie geht's weiter in Görlitz? Schrumpfungsprozesse aus verschiedenen Perspektiven. In: geographie heute 301/302. S. 48-57.

Hemmer, M. (1996): Reisende und Bereiste. Perspektivenwechsel im Tourismus. In: Praxis Geographie 4. S. 18-22.

Hemmer, M. \& R. Uphues (2012): Abwanderung aus der Großwohnsiedlung Berlin-Marzahn. Eine Analyse mittels der vier Raumperspektiven der Geographie. In: Praxis Geographie 1. S. 22-26.

Hennings, W. (1996): Hinsichten auf andere Kulturen. Samoa im Wechsel von Makro- zur Mikroperspektive. In: Praxis Geographie 1996(4). S. 10-13.

Hepp, K. (2017): Auf Spurensuche in der Stadt. Urbane Räume multiperspektivisch entdecken. In: geographie heute 333. S. 17-21.

Hofmann, R., S. Applis \& C. Fritsch (2016): Wie kann ein gerechter Umgang mit Flüchtlingen aussehen? Ein Rollenspiel. In: Praxis Geographie 2. S. 34-39.

Hoogen, A. (2013): Der Bau einer Biogasanlage. Ein Rollenspiel zu einem lokalen Raumkonflikt mit globaler Perspektive. In: Praxis Geographie 4. S. 16-21.

Junker, S. (2011): Warum will Norwegen nicht in die EU? Ein Rollenspiel zur Förderung der Multiperspektivität. In: Praxis Geographie 9. S. 35-39.

Landtwing Blaser, M. \& S. Ziegler (2013): Touristische Resorts in den Alpen - Chancen und Risiken. Das Beispiel Andermatt Swiss Alps aus geographischer und historischer Perspektive. In: Praxis Geographie 9. S. 33-43. 
Mentz, O. (2002): Welcher Tourismus für Nendaz? Der Konflikt Umwelt-Tourismus im Rollenspiel. In: geographie heute 203. S. 22-25.

Rempfler, A. \& M. Landtwing Blaser (2016): Lernaufgabe Benidorm: Massentourismus multiperspektivisch betrachten. In: Geographie aktuell \& Schule 223. S. 26-39.

Reuschenbach, M. (2007): Bergwald ist Schutzwald! Die Bedeutung des Bergwaldes auf Postern und in einem Rollenspiel verdeutlichen. In: geographie heute 251. S. 8-16.

Reuschenbach, M. (2005): Das Gruppenpuzzle als Rollenspiel. Eine Unterrichtsform für die Bewertung der Umweltkatastrophe am Aralsee. In: geographie heute 235. S. 42-44.

Riediger, A. (1997): Nordsee- Erdölförderung oder Umweltschutz? Ein Rollenspiel. In: Praxis Geographie 5. S. 26-29.

Richter J. \& D. Conrad (2017): Rettung des tropischen Regenwaldes im Amazonasgebiet. Perspektivenwechsel einüben. In: Praxis Geographie 5. S. 22-25.

Schramke, W. \& A. Uhlenwinkel (2004): Eukalyptus in Portugal. Perspektivenwechsel im globalen ökologischen Dorf. In: Praxis Geographie 3. S. 27-31.

Schrüfer, G., G. Obermaier \& S. Schwarze (2016): Raumwahrnehmung aus unterschiedlichen Perspektiven am Beispiel Tansania - Empirische Untersuchungen und Konsequenzen für den Geographieunterricht. In: GWUnterricht 142/143. S. 91-101.

Schüssler, F. \& S. Hof (2011): Strom aus der Wüste? DESERTEC: Perspektiven einer Energiepartnerschaft zwischen Afrika und Europa. In: geographie heute 289. S. 22-28.

Sprave, H. (2007): Klimaschutz vs. Ernährungssicherung. Ein Rollenspiel zur aktuellen Diskussion. In: Praxis Geographie 10. S. 30-35.

Wilhelmi, V. (2016): Syrien: Krieg, Hunger, Flucht. Eine mehrperspektivische Annäherung. In: Praxis Geographie 2. S. 22-26.

Wolf, S. (1996): Was heißt denn hier Schlafstadt? Perspektivenwechsel in der Stadtplanung. In: Praxis Geographie 4. S. 24-27.

\section{Literatur}

Arndt, S. (2006): Rassismus und der deutsche Afrikadiskurs. In: Arndt, S. (Hg.): AfrikaBilder. Studien zu Rassismus in Deutschland. Unrast, Münster. S. 9-45

Aschersleben, K. (1993): Welche Bildung brauchen Schüler? Vom Umgang mit dem Unterrichtsstoff. Julius Klinkhardt, Bad Heilbrunn.

Budke, A., A. Creyaufmüller, M. Kuckuck, M. Meyer, F. Schäbitz, K. Schlüter \& G. Weiss (2015): Argumentationsrezeptionskompetenzen im Vergleich der Fächer Geographie, Biologie und Mathematik. In: Budke, A., A. Creyaufmüller, M. Kuckuck, M. Meyer, F. Schäbitz, K. Schlüter \& G. Weiss (Hrsg.): Fachlich argumentieren ler- nen: didaktische Forschungen zur Argumentation in den Unterrichtsfächern. Waxmann, Münster. S. 272-297.

Bauriedl, S. (2016): Politische Ökologie: nicht-deterministische, globale und materielle Dimensionen von Natur/ Gesellschaft-Verhältnissen. In: Geographica Helvetica 71(4). S. 341-351.

Bonacker, T. (2009): Konflikttheorie. In: Kneer, G. \& M. Schroer (Hrsg.): Handbuch Soziologische Theorien. GWV Fachverlage GmbH, Wiesbaden. S. 179- 197.

Bryant, R. L. \& S. Bailey (1997): Third World Political Ecology. Routledge, London.

Dahrendorf, R. (2010): Homo Sociologicus. Ein Versuch zur Geschichte, Bedeutung und Kritik der sozialen Rolle. Verlag für Sozialwissenschaften, Wiesbaden.

DGfG - Deutsche Gesellschaft für Geographie (2017): Bildungsstandards im Fach Geographie für den Mittleren Schulabschluss mit Aufgabenbeispielen. https:// geographie.de/wp-content/uploads/2019/05/Bildungsstandards_Geographie_9.Aufl_.2017.pdf (14.09.2020)

Duncker, L. (2005): Professionalität des Zeigens. Mehrperspektivität als Prinzip der Allgemeinen Didaktik. In: Duncker, L. \& W. Sander (Hrsg.): Perspektivenvielfalt im Unterricht. Surkamp/Kohlhammer, Stuttgart. S. 9-20.

Field, L. \& J. Higley (1983): Eliten und Liberalismus. Westdeutscher Verlag, Opladen.

Frenkel-Brunswik, E. (1949): Intolerance of Ambiguity as an Emotional and Perceptual Personality Variable. In: Journal of Personality 18. S. $108-143$.

Kaminske, V. (2003): Denken in räumlichen Kategorien notwendige Kompetenz? Eine Aufgabe der Geographie. In: Huwendiek, V. \& D. Stöcklin (Hrsg.): Nachdenklichkeit und Nachhaltigkeit - Perspektiven der Lehrerbildung. Festschrift z. 75-jährigen Jubiläum d. Staatl. Seminars f. Schulpädagogik, Karlsruhe. S. 129-138.

Kaminske, V. (2012): Die räumliche Wahrnehmung: Grundlagen für Geographie und Kartographie. Wissenschaftliche Buchgesellschaft, Darmstadt.

Kiehl, C. \& B. Schnerch (2018): Demokratiekompetenzen auf dem Prüfstand - Schule als Erfahrungsraum für Mündigkeit und Ambiguitätstoleranz? In: Institut für Demokratie und Zivilgesellschaft (IDZ) - Thüringer Dokumentations- und Forschungsstelle gegen Menschenfeindlichkeit (Hrsg.): Gesellschaftlicher Zusammenhalt (= Wissen schafft Demokratie 03, Schriftenreihe des Instituts für Demokratie und Zivilgesellschaft). Amadeu Antonio Stiftung, Berlin. S. 111-120.

Kruckemeyer, F. (1995): Reisebilder aus vertrauten fremden Welten. In: Hasse, J. (Hg.): Gefühle als Erkenntnisquelle. Selbstverlag des Instituts für Didaktik der Geographie, Frankfurt am Main. S. 113-148.

Kuckartz, U. (2018): Qualitative Inhaltsanalyse: Methoden, Praxis, Computerunterstützung. Beltz Juventa, Weinheim Basel.

Kuckuck, M. (2014): Konflikte im Raum. Verständnis von gesellschaftlichen Diskursen durch Argumentation im Geographieunterricht. Monsenstein und Vannerdat, Münster. 
Lambert, D. \& J. Morgan (2010): Teaching geography 11-18: a conceptual approach. Open University Press: Maidenhead.

Lehner, M. (2020): Didaktische Reduktion. UTB, Bern.

Lehner, M. (2011): Viel Stoff - wenig Zeit. Wege aus der Vollständigkeitsfalle. Haupt Verlag, Bern.

Lütje, A. \& A. Budke (2021): „Es sind doch Begegnungen, wonach wir suchen". Narration und Emotionalität im Geographieschulbuch. In: GW-Unterricht 161. S. 35-50.

Mayntz, R. \& F. W. Scharpf (1995): Der Ansatz des akteurszentrierten Institutionalismus. In: Mayntz, R. \& F. W. Scharpf (Hrsg.): Gesellschaftliche Selbstregelung und politische Steuerung. Campus-Verlag, Frankfurt/ New York.

Mayring, P. (2015): Qualitative Inhaltsanalyse: Grundlagen und Techniken. Beltz, Weinheim/Basel.

Mitchell, R. K., B. R. Agle \& D. J. Donna (1997): Toward a Theory of Stakeholder Identification and Salience: Defining the Principle of Who and What really Counts. In: Academy of Management Review 22(4). S. 853-886.

Nehrdich, T. (2011): Kontroversität. Neue Herausforderungen für eine aktuelle Geographiedidaktik. GW-Unterricht 124. S. 15-25.

Reuber, P. (2000): Die Politische Geographie als handlungsorientierte und konstruktivistische Teildisziplin - angloamerikanische Theoriekonzepte und aktuelle Forschungsfelder. In: Geographische Zeitschrift 88(1). S. 36-52.

Reuber, P. (2001): Möglichkeiten und Grenzen einer handlungsorientierten Politischen Geographie. In: Reuber, P. \& G. Wolkersdorfer (Hrsg.): Politische Geographie. Handlungsorientierte Ansätze und Critical Geopolitics. S. 77-92.

Reuschenbach, M. (2007): Bergwald ist Schutzwald! Die Bedeutung des Bergwaldes auf Postern und in einem Rollenspiel verdeutlichen. In: geographie heute 251. S. 8-16.

Robbins, P. (2012): Political Ecology. A critical introduction. Willey-Blackwell, Sussex.

Rhode-Jüchtern, T. (2004): Derselbe Himmel, verschiedene Horizonte. Zehn Werkstücke zu einer Geographiedidaktik der Unterscheidung. Institut für Geographie und Regionalforschung der Universität, Wien.
Rhode-Jüchtern, T. (2011): Diktat der Standardisierung oder didaktisches Potenzial? - Die Bildungsstandards Geographie praktisch denken. In: GW-Unterricht 124. S. 3-14.

Rhode-Jüchtern, T. (2015): Kreative Geographie. Bausteine zur Geographie und ihrer Didaktik. Wochenschau Verlag, Schwalbach.

Scharpf, F. W. (2000): Interaktionsformen: akteurzentrierter Institutionalismus in der Politikforschung. UTB, Opladen.

Schimank, S. (2011): So viel zu Akteuren! Ein Minimalkonzept zur Beantwortung einer Vorfrage soziologischer Erklärungen. In: Lüdtke, N. \& H. Matsuzaki (Hrsg.): Akteur - Individuum - Subjekt. Fragen zu ,Personalität und ,Sozialität'. Verlag für Sozialwissenschaften, Wiesbaden. S. 23-44.

Schmidt-Wulffen, W. (1997): Jugendliche und „Dritte Welt": Bewusstsein, Wissen und Interessen. In: GWUnterricht 66. S. 11-20.

Stöber, G. (2011): Zwischen Wissen, Urteilen und Handeln - „Konflikt“ als Thema im Geographieschulbuch. In: Meyer, C., R. Henrÿ \& G. Stöber (Hrsg.): Geographische Bildung. Kompetenzen in didaktischer Forschung und Schulpraxis (Tagungsband zum HGD-Symposium in Braunschweig). (= Geographiedidaktische Forschungen). Braunschweig. S. 68-81.

Tröger, S. (1993): Das Afrikabild bei deutschen Schülerinnen und Schülern. Breitenbach Publishers, Saarbrücken.

Vogel, P. (1995): Didaktische Reduktion. In: Haller, H. \& H. Meyer (Hrsg.): Enzyklopädie Erziehungswissenschaft, Ziele und Inhalte der Erziehung und des Unterrichts. Klett-Cotta, Stuttgart/Dresden. S. 567-571.

Weber, M. (1980): Wirtschaft und Gesellschaft. Grundriß einer verstehenden Soziologie. Mohr Siebeck, Tübingen.

Wehling, H. G. (1977): Konsens à la Beutelsbach? Nachlese zu einem Expertengespräch. In: Schiele, S. \& H. Schneider (Hrsg.): Das Konsensproblem in der politischen Bildung. Ernst Klett, Stuttgart. S. $173-184$.

World Economic Forum (2017): The Global Risks Report 2017. http://www3.weforum.org/docs/GRR17_Report_ web.pdf (17.08.2020) 